\title{
Targeting Notch-activated M1 macrophages attenuate lung tissue damage in a rat model of ventilator induced lung injury
}

\author{
DANPING YIN $^{1}$, WEIMING WANG $^{2}$, WEI HAN $^{3}$ and CHEN FAN ${ }^{4}$ \\ ${ }^{1}$ Department of Disease Prevention and Control, No. 960 Hospital of PLA, Jinan, Shandong 250031; \\ ${ }^{2}$ Electrocardiogram Room, Yantai Yuhuangding Hospital Affiliated to Qingdao University, Yantai, Shandong 264001; \\ Departments of ${ }^{3}$ Training and ${ }^{4}$ Laboratory Diagnosis, No. 960 Hospital of PLA, Jinan, Shandong 250031, P.R. China
}

Received September 4, 2018; Accepted June 27, 2019

DOI: $10.3892 /$ ijmm.2019.4315

\begin{abstract}
Ventilator induced lung injury (VILI) may be involved in the activation of alveolar macrophages. The purpose of this study was to investigate the relationship between the Notch signaling pathway and macrophage polarization in VILI. The VILI model was established using rats. Hematoxylin-eosin staining was used to test the lung tissue morphology. Bicinchoninic acid assay and ELISA were performed to detect protein and tumor necrosis factor (TNF)- $\alpha$, interleukin (IL)-6, IL-10 levels in bronchoalveolar lavage fluids (BALF), respectively. The ratio of alveolar M1 and M2 macrophages was detected by flow cytometry. The mRNA and protein expression levels of Notch pathway-related proteins were determined using reverse transcription-quantitative PCR and western blotting. The present study found that high-frequency mechanical ventilation could cause pulmonary edema and increase the levels of protein, TNF- $\alpha$ and IL-6 in BALF while decreasing the level of IL-10 in BALF. High-frequency mechanical ventilation also induced polarization of alveolar macrophages to M1. The results also showed a significant increase in the levels of Notch pathway-related proteins including notch intracellular domain, Hes1, Hes5 and Heyl. Injection of N-[N-(3,5-difluorophenylacetyl)-1-alanyl] phenylglycine t-butyl ester could inhibit the Notch pathway and such an inhibition protected lung tissue and reduced lung inflammation caused by mechanical ventilation. After the Notch pathway was inhibited, the level of M1 polarization of macrophages caused by high-frequency mechanical ventilation was reduced. VILI caused pulmonary inflammation and macrophages to polarize to M1 and upregulated
\end{abstract}

Correspondence to: Dr Danping Yin, Department of Disease Prevention and Control, No. 960 Hospital of PLA, 25 Shifan Road, Tianqiao, Jinan, Shandong 250031, P.R. China

E-mail: danpingy_yindp@163.com

Key words: high frequency mechanical ventilation, ventilator induced lung injury, macrophages, Notch pathway the expression levels of Notch pathway-related proteins. The inhibition of Notch pathway also reduced the proportion of M1 macrophages and inflammatory responses.

\section{Introduction}

Using mechanical devices, mechanical ventilation replaces or controls autonomous breathing movements (1). It is an indispensable part of general anesthesia and one of the main methods in treating respiratory failure (2). However, studies found that the incidence of ventilator-induced lung injury (VILI) was high, which seriously affected the health and quality of life of patients (3-5). The prevention and mitigation of the occurrence of VILI have also attracted much research attention.

At present, the pathogenesis of VILI still remains unclear. A previous study has suggested that VILI was mainly caused by mechanical injury (6). It was discovered that mechanical damage could act on signal transduction in effector cells, thereby affecting the expression of target genes and subsequently cell apoptosis, damage, migration or various phenotypic changes, which triggered a series of inflammatory responses, cascades of inflammatory mediators and caused inflammatory lesions in the lungs. Previous studies have shown that VILI could cause the release of inflammatory factors such as tumor necrosis factor (TNF- $\alpha$ ) and interleukin (IL-6), aggravating lung injury (7-9).

In the process of lung inflammation, the body's innate immune system plays an important role. Alveolar macrophages are lung-resident cells (10). Macrophages can be divided into 2 major subtypes, that is, $\mathrm{Ml}$ (pro-inflammatory) and M2 (anti-inflammatory) (11). M1 type macrophages induce secretion of TNF- $\alpha$ and promote inflammation, while M2 type macrophages have anti-inflammatory effects and promote tissue repair $(12,13)$. A previous study found that mechanical stretch stimulation can activate macrophages and macrophages recruit peripheral neutrophils to the lungs and participate in the inflammatory response when activated (14). Another study showed that alveolar macrophages played an important regulatory role in the mechanism of VILI production (15). However, the 
mechanism of activation and regulation of alveolar macrophage activation in mechanical ventilation has not been fully elucidated.

Notch receptors are mainly distributed on the surface of stem cells or protocells and are also widely expressed on the surface of various immune cells such as macrophages, $\mathrm{T}$ cells and dendritic cells (16). A previous study has suggested that the activation of Notch signaling pathway would cause macrophage polarization and promote the secretion of IL-6 (17). Han et al (18) have shown that blocking the Notch signaling pathway could reduce the inflammatory response and tissue damage. It has also been found that inhibiting the Notch signaling pathway could reduce the expression of TNF- $\alpha$ (19). In addition, the use of the Notch signaling pathway inhibitor $\mathrm{N}$-[N-(3,5-difluorophenylacetyl)-1-alanyl] phenylglycine t-butyl ester (DAPT) can improve arthritis symptoms and joints in arthritic damaged mice $(20,21)$. Therefore, the present study hypothesized that the Notch signaling pathway was involved in the regulation of pulmonary inflammatory response after mechanical ventilation and participation in the occurrence and development of VILI.

The present study mainly investigated the relationship between VILI and Notch signaling pathways in regulating macrophage polarization and studied the pathogenesis of VILI. The present findings provide evidence for and new approaches to VILI prevention and treatment.

\section{Materials and methods}

Animals and establishment of mechanical ventilation lung injury model. A total of 60 male Sprague-Dawley rats (weight: 250-300 g; age: 8 weeks) were purchased from the Laboratory Animal Center. Modeling and follow-up experimental programs had been approved by the Institutional Animal Care and Use Committee and China Council on Animal Care. The rats were fed with a normal diet with water ad libitum before the treatment and kept on a 12-h light/dark cycle in a controlled room at $22 \pm 2^{\circ} \mathrm{C}$ temperature in $60-70 \%$ humidity. The rats were fasted $12 \mathrm{~h}$ before surgery but were allowed to have free access to drinking water. The rats were anesthetized with an intraperitoneal injection of $10 \%$ chloral hydrate $300 \mathrm{mg} / \mathrm{kg}$ in a supine position and fixed on an adjustable warming pad (Shanghai Alcott Biotech, Co., Ltd.), and the body temperature of the rats was maintained at $37 \pm 1^{\circ} \mathrm{C}$. Peritonitis was not observed in rats after anesthesia. After anesthesia, the trachea was exposed by cutting along the midline and the rats were subjected to tracheal intubation. Ophthalmic scissors were used to cut a small opening between the tracheal cartilage and a 22-gauge sterile intravenous indwelling needle cannula was inserted slowly. The casing was fixed with surgical wire and connected to a small animal ventilator (Inspira ASV; Harvard Apparatus, Ltd.). The tidal volume was adjusted according to the weight, with reference to previous studies $(22,23)$. The tidal volume parameter of the normal tidal volume group (LVT group; $n=10$ ) was $8 \mathrm{ml} / \mathrm{kg}$ and in the high tidal volume group (HVT; $\mathrm{n}=10$ ) it was $40 \mathrm{ml} / \mathrm{kg}$. The rats in the control group (Con; $\mathrm{n}=10$ ) were subjected to a tracheotomy and tracheal intubation, and spontaneous breathing was retained. The ventilator parameters was as follows: The inspiration and expiration ratio was 1:2, the respiratory rate was 80 times/min, the oxygen concentration was $40 \%$, and the positive end-expiratory pressure was 0 . The high frequency oscillating ventilation time was $4 \mathrm{~h}$. The rats in each group were alive during mechanical ventilation.

Other rats were modeled by the same method and divided into the Con, HVT and DAPT groups. The DAPT group received intraperitoneal injection of DAPT $100 \mathrm{mg} / \mathrm{kg} 3 \mathrm{~h}$ before mechanical ventilation, while the other groups received the same dose of DMSO.

Humane endpoints were defined in case of unexpected or major change in the behavior (quantified using a scoring system). In short, the endpoints included: Weight loss $>15 \%$ between two adjacent body weighing sessions (three times a week); Signs of severe dyspnea, defined as an inability of the rats to keep oxygen saturation $>90 \%$; Signs indicating encephalitis, e.g., nystagmus, paresis, or head tilt.

Hematoxylin and eosin $(H \& E)$ staining. H\&E staining was applied to observe the lung tissues in each group. After modeling, lung tissues from rats were immediately fixed in formalin for $24 \mathrm{~h}$ at room temperature and were then dehydrated with alcohol and embedded in paraffin. The sample was cut into $4-\mu \mathrm{m}$ thick-uniform flakes and placed on APES-coated glass slides. The sections were deparaffinized using xylene and hydrated and stained with the H\&E reagent (Sigma-Aldrich; Merck KGaA). Hematoxylin was added and the sample was incubated at room temperature for $5 \mathrm{~min}$. After washing, eosin was added and the sample was incubated at room temperature for $\sim 2 \mathrm{~min}$. After being washed with gradient ethanol, a neutral gel was used for sealing. The alveolar morphology was observed under a light microscope and assessed by the lung injury score.

Lung wet/dry ratio. All rats were sacrificed by intraperitoneal injection of sodium pentobarbital $(200 \mathrm{mg} / \mathrm{kg})$. The sound of breathing and heartbeat (respiratory arrest and cardiac arrest) could not be heard through the stethoscope and this confirmed the death of the rats. After the rats were sacrificed, the pulmonary circulation was washed with PBS and the left lung was separated and weighed as the wet lung weight. The wet lungs were placed in a $65^{\circ} \mathrm{C}$ oven and allowed to be dried for $48 \mathrm{~h}$ and weighed again as the dry lung weight. The lung wet/dry ratio=wet lung weight/dry lung weight.

Bicinchoninic acid (BCA) assay. The protein concentration in the bronchoalveolar lavage fluids (BALF) was measured using the BCA assay. After the rats were sacrificed, PBS was injected into the lungs via the trachea, the liquid was aspirated slowly and the procedure was repeated 3 times. The supernatant of the aspirated liquid was separated by centrifugation at $12,000 \mathrm{x} \mathrm{g}$ for $10 \mathrm{~min}$ at $4^{\circ} \mathrm{C}$ and the protein concentration in the supernatant was measured. BCA kit was purchased from Biomiga, Inc., and the reagents were added according to the manufacturer's protocol. Absorbance at $570 \mathrm{~nm}$ was measured by a microplate reader (FilterMax F3/F5; Molecular devices, LLC) and the protein concentrations were calculated from the standard curve concentration.

ELISA. The concentrations of TNF- $\alpha$ (CSB-E11987r), IL-6 (CSB-E04640r) and IL-10 (CSB-E04595r) in BALF were measured by ELISA. The kit was purchased from Cusabio Biotech 
Table I. The sequences of primers.

\begin{tabular}{lll} 
Gene & \multicolumn{1}{c}{ Forward (5'-3') } & \multicolumn{1}{c}{ Reverse (5'-3') } \\
\hline NICD & TGGCCTCAATGGATACAAATG & GGGCCAACACCACCTCAC \\
Hes-1 & CCAGCCAGTGTCAACACGA & AATGCCGGGAGCTATCTTTCT \\
Hes-5 & AGTCCCAAGGAGAAAAACCGA & GCTGTGTTTCAGGTAGCTGAC \\
Hey 1 & AAAGACGGAGAGGCATCATCG & GCAGTGTGCAGCATTTTCAGG \\
GAPDH & CCTTCCGTGTTCCTACCCC & GCCCAGGATGCCCTTTAGTG
\end{tabular}

NICD, Notch intracellular domain.

Co., Ltd. The enzyme labeling reagent and developer were added following the protocol, and the reaction was terminated by adding a stop solution. Optical densities at $450 \mathrm{~nm}$ were determined using an ELISA reader (Model 680; Bio-Rad Laboratories, Inc.).

Flow cytometry. Flow cytometry was used to detect the polarity of macrophages in lung tissues and the stain buffer (BSA) was purchased from BD Pharmingen; Becton, Dickson and Company. Lung tissues were lysed in a lysis buffer containing $10 \mathrm{mM}$ Tris- $\mathrm{Cl}, 160 \mathrm{mM} \mathrm{KCl}$ and $1.5 \mathrm{mM} \mathrm{MgCl}$, $250 \mathrm{mM}$ sucrose, $0.5 \%$ Nonidet P 40 Substitute (Fluka), $3 \mathrm{mM}$ $\beta$-mercaptoethanol, $2 \mathrm{mM}$ phenylmethanesulfonylflouride, $10 \mu \mathrm{g} / \mathrm{ml}$ leupeptin and $10 \mu \mathrm{g} / \mathrm{ml}$ aprotinin, and the cells were resuspended to a concentration of $4 \times 10^{5}$ cells $/ \mathrm{ml}$. F4/80, Cmaf and inducible nitric oxide synthase (iNOS) antibodies (eBioscience; Thermo Fisher Scientific, Inc.) were added, respectively. The samples were incubated at room temperature in the dark for $10 \mathrm{~min}$. A flow cytometer (Becton, Dickson and Company) was applied to analyze the cell apoptosis, and the data was analyzed using BD CellQuest ${ }^{\mathrm{TM}}$ Pro version 1.2 software (BD Biosciences).

Reverse transcription-quantitative polymerase chain reaction $(R T-q P C R)$. The mRNA expression levels of Notch intracellular domain (NICD), Hes1, Hes5 and Heyl were determined by RT-qPCR. Total RNA was extracted using the TRIzol reagent (Invitrogen; Thermo Fisher Scientific, Inc.) and the reverse transcription kit (Invitrogen; Thermo Fisher Scientific, Inc.) was used to synthesize cDNA at $37^{\circ} \mathrm{C}$ for $15 \mathrm{~min}$, followed by a reverse transcriptase inactivation at $85^{\circ} \mathrm{C}$ for $15 \mathrm{sec}$. RT-qPCR was carried out to determine NICD, Hes1, Hes5 and Heyl, and the amplification reaction procedures were as follows: At $95^{\circ} \mathrm{C}$ for $5 \mathrm{~min}$, followed by 40 cycles $\left(\right.$ at $95^{\circ} \mathrm{C}$ for $15 \mathrm{sec}$, at $60^{\circ} \mathrm{C}$ for $1 \mathrm{~min}$, at $72^{\circ} \mathrm{C}$ for $1 \mathrm{~min}$ ) and a final extension at $72^{\circ} \mathrm{C}$ for $10 \mathrm{~min}$ and held at $4^{\circ} \mathrm{C}$. All primers were obtained from Takara Bio, Inc. and are listed in Table I. GAPDH was used as reference gene. The formula $2^{-\Delta \Delta C q}(24)$ was implemented to analyze the mRNA expression levels.

Western blotting. Western blotting was applied to detect Notch signaling pathway-associated proteins, which included NICD, Hes1, Hes5 and Hey1. The cells were lysed with RIPA (Abcam) and the supernatant was collected by a centrifugation at $12,000 \mathrm{x} \mathrm{g}$ at $4^{\circ} \mathrm{C}$ for $15 \mathrm{~min}$. A BCA assay was used to determine the protein concentration. Protein lysates $(25 \mu \mathrm{g} / \mathrm{lane})$ were separated by $12 \%$ SDS-PAGE. The PVDF membrane
(Bio-Rad Laboratories, Inc.) was transferred by a Trans-Blot Transfer Slot (Bio-Rad Laboratories, Inc.) and blocked with $5 \%$ fat-free milk for $2 \mathrm{~h}$ at room temperature. The primary antibodies (anti-NICD; Abcam; cat. no. ab8925; 1:800; anti-Hes1; Abcam; cat. no. ab71559; 1:700; anti-Hes5; Abcam; cat. no. ab25374; 1:600; anti-Hey1; Abcam; cat. no. ab22614; 1:800) were added following the kit protocol and shaken at room temperature for $2 \mathrm{~h}$ and then incubated at $4^{\circ} \mathrm{C}$ for $12 \mathrm{~h}$. The HRP-conjugated secondary antibodies (rabbit anti-human IgG; Abcam; cat. no. ab6759; 1:8,000; rabbit anti-goat IgG; Abcam; cat. no. ab6741; 1:10,000; goat anti-rabbit IgG; Abcam, ab6721; 1:8,000) were added and incubated at room temperature for $15 \mathrm{~h}$. Chemiluminescence detection was carried out using ECL reagent (SignalFire, cat. no. 6883, Cell Signaling Technology, Inc.). Densitometry was performed using Quantity One software version 2.4 (Bio-Rad Laboratories, Inc.)

Statistical analyses. Data from three independent and repetitive experiments were shown as the mean \pm standard deviation. Differences between the experimental groups were assessed by analysis of variance, followed by Dunnett's post hoc test. Differences were analyzed by GraphPad Prism 6 (GraphPad Software, Inc.). P $<0.05$ was considered to indicate a statistically significant difference.

\section{Results}

Effects of mechanical ventilation on lung tissue. The H\&E staining results showed that the alveolar morphology of the lung tissue from the Con group was normal and there was no inflammatory cell infiltration. The alveolar structure of the LVT group was normal and a mild inflammatory infiltration was observed. In the HVT group, the alveolar structure was disordered, the alveolar space was thickened and a large number of inflammatory cells infiltrated in the lung tissue, and the interstitial hemorrhage showed changes in congestion and hemorrhage and the lung injury score significantly increased $(\mathrm{P}<0.01$; Fig. 1).

Effects of mechanical ventilation on lung and inflammatory factors in $B A L F$. To investigate the effects of mechanical ventilation on pulmonary edema and the inflammatory response in lung tissue, lung wet/dry ratio, protein, TNF- $\alpha$, IL- 6 and IL-10 in BALF were determined. The results showed no difference in lung wet/dry ratio between the Con group and the LVT group, and that the lung wet/dry ratio of the HVT group was 

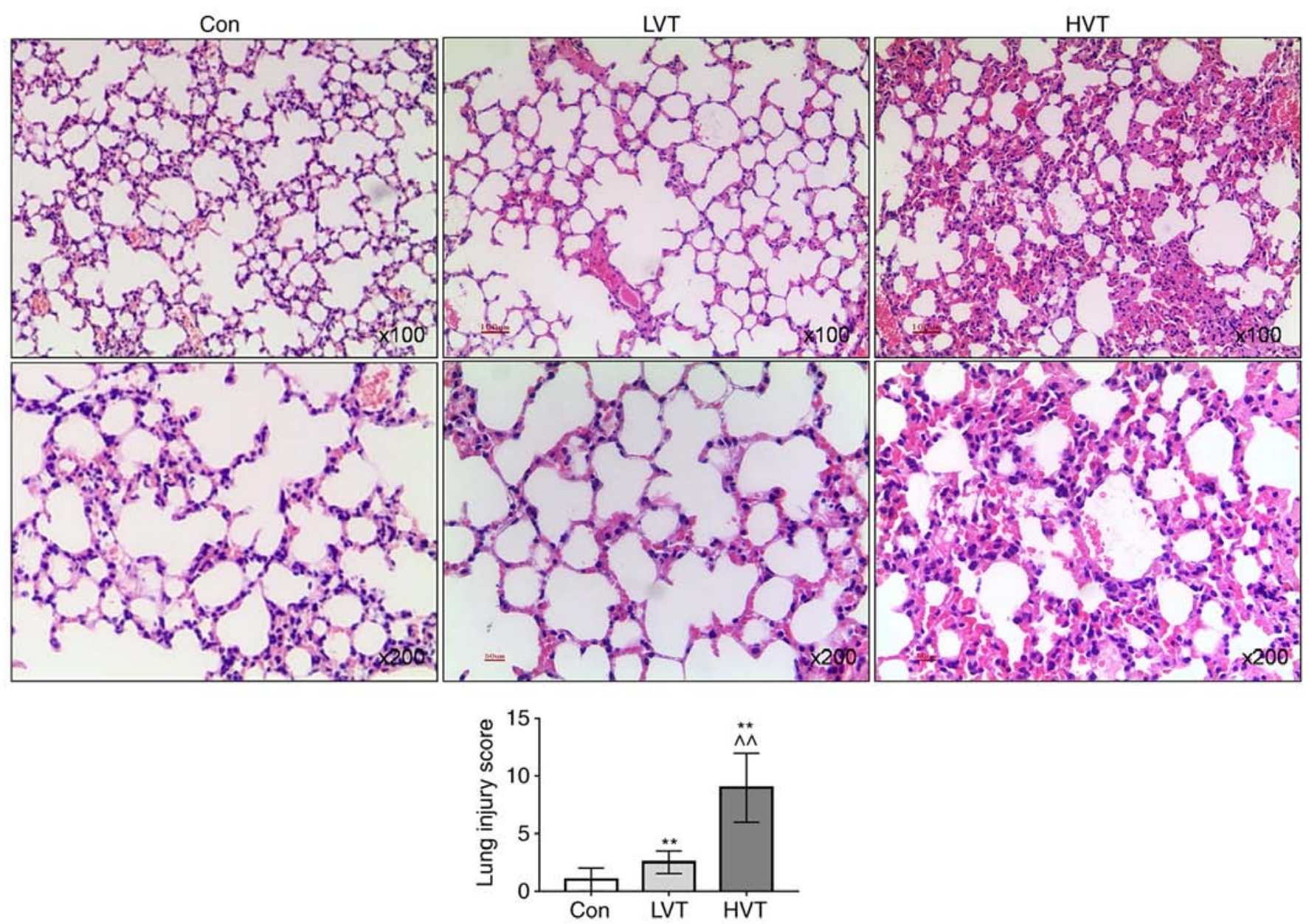

Figure 1. Effects of mechanical ventilation on rat lung tissue. Hematoxylin and eosin staining was used to detect lung tissue morphology in the Con group, LVT group and HVT group. The degree of lung injury was assessed using lung injury score. ${ }^{* *} \mathrm{P}<0.01$ vs. Con group; ${ }^{\wedge} \mathrm{P}<0.01 \mathrm{vs.} \mathrm{LVT} \mathrm{group.} \mathrm{LVT,} \mathrm{normal} \mathrm{tidal}$ volume; HVT, high volume tidal; Con, control.

increased significantly ( $\mathrm{P}<0.05 ;$ Fig. 2A). No significant difference was detected in protein content, TNF- $\alpha$, IL-6 and IL-10 levels in BALF between the Con group and LVT group. The protein content, TNF- $\alpha$ and IL-6 in BALF in HVT group was significantly increased compared with the Con group and the LVT group $(\mathrm{P}<0.01)$, and the IL-10 level in BALF in HVT group was significantly decreased compared with the Con group and the LVT group $(\mathrm{P}<0.01$; Fig. 2B-E). This suggested that high-frequency mechanical ventilation could damage lung tissue and lead to pulmonary edema inflammatory reactions in the lungs.

Effects of mechanical ventilation on the polarization of macrophages. To explore the causes to inflammatory reactions in the lungs, flow cytometry was used to detect the polarization levels of macrophages. The results showed no significant change in M1 macrophage levels in the Con group and those in the LVT group, and that the proportion of M1 macrophages in the HVT group was significantly increased compared with the Con group and the LVT group $(\mathrm{P}<0.01$; Fig. $2 \mathrm{~F})$. For M2 macrophages, the proportions of M2 macrophages in the LVT group and the HVT group were decreased, and the level of M2 macrophages in the HVT group was decreased compared with the LVT group (Fig. 2G), suggesting that mechanical ventilation might induce polarization of macrophages in lung tissue to M1.
Effects of mechanical ventilation on the expression of Notch pathway associated proteins. NICD, Hes1, Hes5 and Hey1 mRNA and protein expression levels were determined by RT-qPCR and western blotting to explore the mechanism of macrophage polarization. The results showed no significant difference in the mRNA and protein expression levels of the 4 proteins between the control group and the LVT group. The mRNA and protein expression levels of NICD, Hes1, Hes5 and Hey1 in HVT group were significantly increased compared with the Con group and the LVT group $(\mathrm{P}<0.01$; Fig. 3A and B). This suggested that high-frequency mechanical ventilation could promote the expression of Notch signaling pathway-associated proteins.

Effects of inhibition of Notch pathway on lung injury in rats. To explore the effects of inhibition of the Notch pathway on lung injury caused by mechanical ventilation, the Notch pathway was inhibited using DAPT. The H\&E staining results showed that the alveolar morphology of the lung tissue in the Con group was normal. In the HVT group, the alveolar structure was disordered, the alveolar space was significantly thickened and a large number of inflammatory cells infiltrated in the lung tissue and the interstitial hemorrhage showed changes in congestion and hemorrhage. The alveolar structure of the DAPT group was basically normal, however, some 

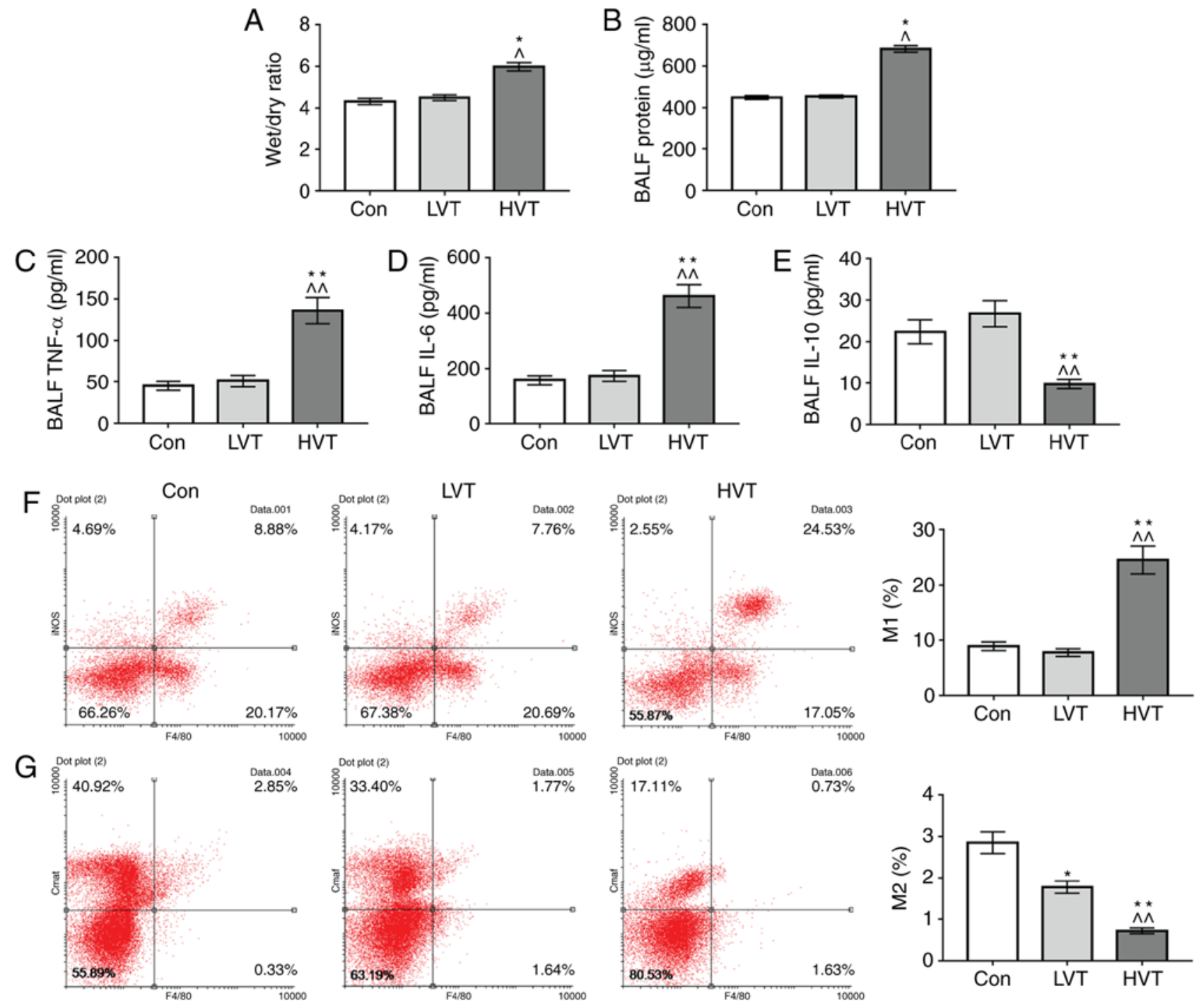

Figure 2. Effects of mechanical ventilation on wet/dry ratio inflammatory factors in BALF and macrophage polarization. (A) The lung wet/dry ratios of the Con group, LVT group and HVT group were weighed and compared. A bicinchoninic acid assay and ELISA were performed to detect (B) protein and (C) TNF- $\alpha$, (D) IL-6, (E) IL-10 levels in BALF respectively. Alveolar macrophage polarization [(F) M1 and (G) M2] was detected using flow cytometry. ${ }^{*} \mathrm{P}<0.05$ and ${ }^{* *} \mathrm{P}<0.01$ vs. Con group; ${ }^{\wedge} \mathrm{P}<0.05$ and ${ }^{\wedge} \mathrm{P}<0.01$ vs. LVT group. $\mathrm{LVT}$, normal tidal volume; HVT, high volume tidal; Con, control; BALF, broncho alveolar lavage fluid; IL, interleukin; TNF, tumor necrosis factor.

inflammatory infiltration occurred. The lung injury score of the DAPT group declined significantly $(\mathrm{P}<0.01 ;$ Fig. 4).

Effects of inhibition of Notch pathway on lung and inflammatory factors in $B A L F$. To investigate the effects of inhibition of Notch pathway on pulmonary edema and the inflammatory response in lung tissue, lung wet/dry ratio, protein, TNF- $\alpha$, IL-6 and IL-10 in BALF were determined. The results showed that lung wet/dry ratio, protein content, TNF- $\alpha$ and IL-6 levels in BALF in the HVT group were increased compared with the Con group and DAPT group, and that no significant difference on above indicators between the Con group and the DAPT group was observed. The IL-10 levels in the HVT group and DAPT group were decreased compared with the Con group, however, the IL-10 level in the DAPT group was increased compared with the HVT group (Fig. 5A-E). This suggested that the inhibition of the Notch pathway by DAPT could attenuate lung injury and the inflammatory response in the lungs of mechanical ventilation.
Effects of inhibition of Notch pathway on the polarization of macrophages. The results showed that the proportions of M1 macrophages in the HVT group and DAPT group were significantly increased compared with the Con group $(\mathrm{P}<0.01)$, while the proportion of M1 macrophages in DAPT group was significantly decreased compared with in the HVT group $(\mathrm{P}<0.01)$. The proportions of M2 macrophages in the HVT group and DAPT group were decreased compared with the Con group, while the proportion of M2 macrophages in DAPT group was significantly increased compared with the HVT group $(\mathrm{P}<0.01$; Fig. 5F and $\mathrm{G})$. This suggested that the inhibition of Notch pathway by DAPT could downregulate macrophage M1 polarization caused by mechanical ventilation.

Inhibition of Notch pathway by DAPT. To demonstrate the inhibitory effect of DAPT on Notch pathway, RT-qPCR and western blotting were performed to detect NICD, Hes1, Hes5 and Hey1 mRNA and protein expression levels in each group. The results showed that the NICD, Hes1, Hes5 and Hey1 
A NICD Hes-1 Hes-5 Hey1


Figure 3. Effects of mechanical ventilation on the Notch pathway of macrophages. (A) The mRNA expression of the Notch signaling pathway-related proteins NICD, Hes1, Hes5 and Hey1 in three groups were detected using reverse transcription-quantitative PCR. (B) The protein expression of the NICD, Hes1, Hes5 and Hey1 in 3 groups were detected using western blotting. ${ }^{* *} \mathrm{P}<0.01$ vs. Con group; ${ }^{\wedge} \mathrm{P}<0.01$ vs. LVT group. LVT, normal tidal volume; HVT, high volume tidal; Con, control; NICD, Notch intracellular domain.

mRNA and protein expression levels in the DAPT group were decreased compared with the HVT group (Fig. 6A and B), indicating that using DAPT could inhibit the levels of Notch pathway-related protein.

\section{Discussion}

Excessive alveolar expansion or excessive intrapulmonary pressure caused by mechanical ventilation can lead to damage to lung tissue, interstitial structures and alveolar membrane damage, and such damage is characterized by pulmonary edema and oxygen dysfunction (25). The main pathological change in VILI is the increased permeability of the alveolar capillary membrane, which is a result of the release of inflammatory cells and inflammatory factors. As demonstrated by previous studies, the immunoinflammatory response caused by an early activation of macrophages was found in VILI $(26,27)$.

Previous studies have found that high-frequency mechanical ventilation led to elevated levels of TNF- $\alpha$ and IL- 6 in serum and lung BALF $(27,28)$. To investigate the effects of mechanical ventilation on lung and alveolar macrophages, rats were used to establish a mechanical ventilation model and detected changes in lung tissue and in inflammatory factors in BALF. The results showed that high-frequency mechanical 

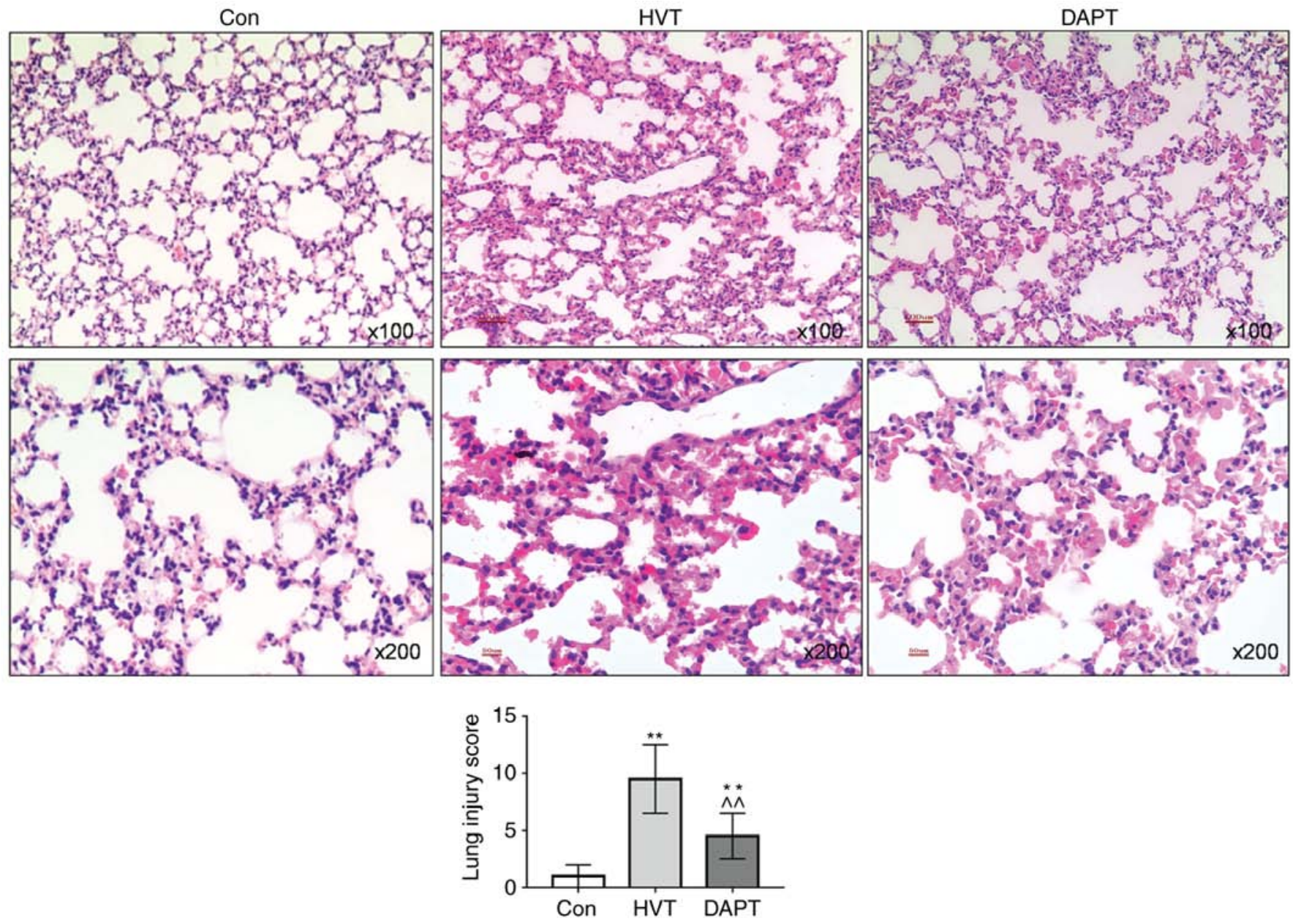

Figure 4. Effects of DAPT on rat lung tissue. Hematoxylin and eosin staining was used to detect lung tissue morphology in the Con group, HVT group and DAPT group, respectively. The degree of lung injury was assessed using lung injury score. ${ }^{* *} \mathrm{P}<0.01$ vs. Con group; ${ }^{\wedge} \mathrm{P}<0.01$ vs. HVT group. LVT, normal tidal volume; HVT, high volume tidal; Con, control; DAPT, N-[N-(3,5-difluorophenylacetyl)-1-alanyl] phenylglycine t-butyl ester.

ventilation changed the alveolar structure and caused inflammatory cell infiltration and pulmonary edema, suggesting that the VILI rat model was successfully established. The results also showed that high-frequency mechanical ventilation also increased the protein content, TNF- $\alpha$ and IL-6 levels in BALF, and decreased the IL-10 level, therefore causing an inflammatory response. It has been shown that the release of inflammatory factors was not only secondary to non-cell damage, but also could be induced by mechanical ventilation, which induces macrophage activation, recruits inflammatory cells to the lungs and amplifies the inflammatory response (29-31).

In order to study the causes of lung inflammation in VILI rats, the polarization of macrophages in lung tissue was measured. The results showed that high-frequency mechanical ventilation caused polarization of rat macrophages to M1. In previous years, a study also found abnormal polarization of alveolar macrophages in VILI animals, moreover, TNF- $\alpha$ also induces macrophage polarization to M1 and M1 macrophages release TNF- $\alpha$ and IL- 6 to aggravate the inflammatory response (13). In addition, macrophage polarization will also recruit a large number of peripheral neutrophils to the lungs, aggravating inflammatory lesions of the lungs $(15,32)$. This suggested that high-frequency mechanical ventilation could aggravate lung injury in VILI rats by inducing polarization of macrophages to M1.

To investigate the mechanism of polarization of macrophages in VILI rats, the levels of Notch pathway-associated proteins were examined in rat macrophages. The results showed that high-frequency mechanical ventilation significantly upregulated NICD, Hes1, Hes5 and Hey1 mRNA and protein expression levels. The Notch signaling pathway plays an important role in immune regulation (33). After the Notch receptor binds to the ligand, the extracellular fragment is released and the rest is cleaved by $\gamma$-secretase and releases NICD (34). NICD binds to the recombination signal binding protein $\mathrm{J}$ in the nucleus to form transcriptional activators, which affects the expression of the target genes Hes1, Hes5 and Hey1. Hes1, Hes5 and Hey1 gene expression products interact with other signaling pathways to affect cell proliferation, differentiation and apoptosis (35-38). A previous study has shown that upregulation of macrophage Notch-1 gene expression level could promote macrophage activation and activated Notch signaling pathway can affect mature macrophage function by upregulating levels of interferon (IFN) $-\gamma$ (38-40). Wongchana et al (41) also demonstrated that LPS and IFN- $\gamma$ could stimulate macrophage expression of NICD-1, while NICD-1 can promote the secretion of IL-6 
A

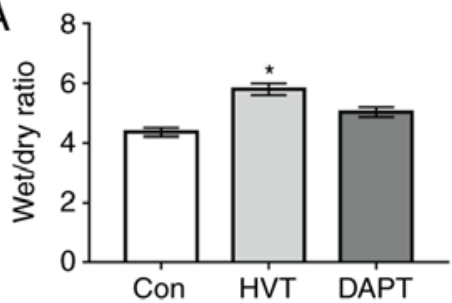

$\mathrm{B}$



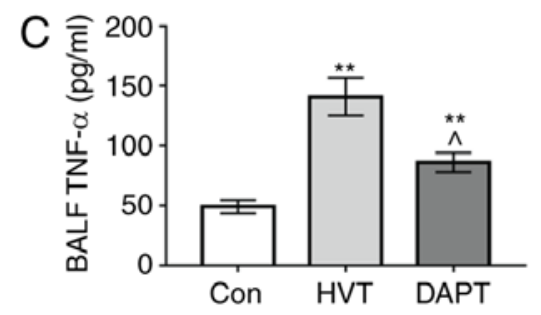
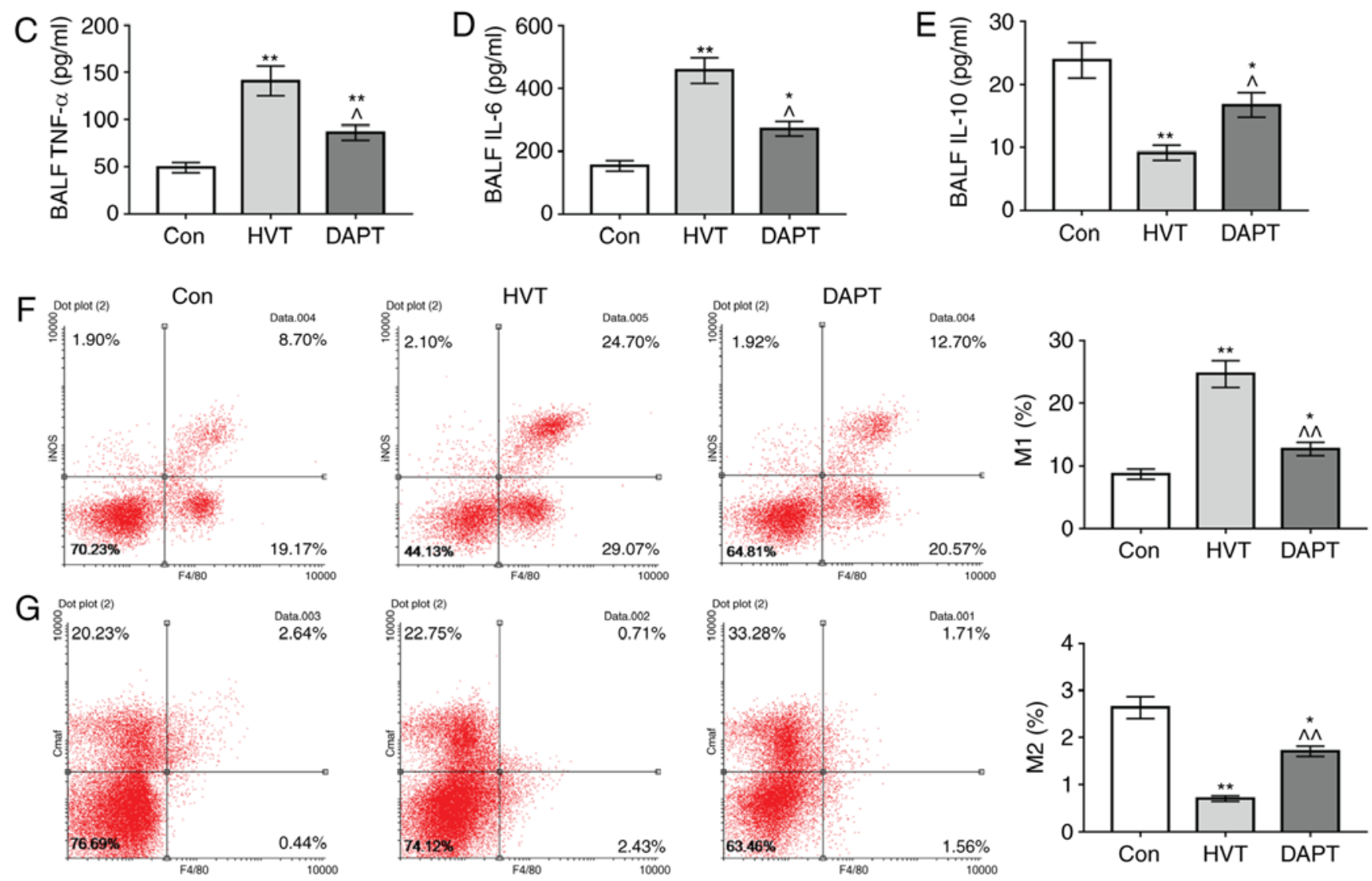

Figure 5. Effects of DAPT on wet/dry ratio inflammatory factors in BALF and macrophage polarization. (A) The lung wet/dry ratios of the Con group, HVT group and DAPT group were weighed and compared. BCA assay and ELISA were used to test (B) protein, (C) TNF- $\alpha$, (D) IL-6 and (E) IL-10 levels in BALF, respectively. Alveolar macrophage polarization $[(\mathrm{F}) \mathrm{M} 1$ and $(\mathrm{G}) \mathrm{M} 2]$ was detected by flow cytometry. ${ }^{*} \mathrm{P}<0.05$ and ${ }^{* *} \mathrm{P}<0.01$ vs. control group; ${ }^{\wedge} \mathrm{P}<0.05$ and ${ }^{\wedge} \mathrm{P}<0.01$ vs. HVT group. LVT, normal tidal volume; HVT, high volume tidal; Con, control; DAPT, N-[N-(3,5-difluorophenylacetyl)-1-alanyl] phenylglycine t-butyl ester; BALF, broncho alveolar lavage fluid; IL, interleukin; TNF, tumor necrosis factor.

via the nuclear factor- $\kappa \mathrm{B}$ pathway to induce macrophage polarization to M1. The results of this study showed that the NICD, Hes1, Hes5 and Hey1 protein expression levels were increased in the lung macrophages of the HVT group, indicating that high-frequency mechanical ventilation promoted the activation of the Notch pathway, thereby inducing macrophages to polarize to M1 and aggravating the inflammatory response.

To further explore the mechanism of macrophage polarization in VILI rats, the effect of the Notch pathway on macrophage polarization was investigated. A previous study found that subcutaneous injection of DAPT effectively inhibited the activity of the Notch pathway and that DAPT lowered the level of NICD (18). In addition, a different study also found that DAPT did not affect the level of TNF- $\alpha$ in arthritic mice (42). The results of the present study showed that the macrophage NICD, Hes1, Hes 5 and Hey1 mRNA and protein expression levels in the lung tissue of DAPT mice were decreased, suggesting that the Notch signaling pathway in the DAPT group was successfully inhibited by intraperitoneal injection of DAPT. The results of this study also showed that inhibition of the Notch pathway could reduce pulmonary edema and pulmonary inflammatory factor release caused by high-frequency mechanical ventilation and could inhibit the polarization of macrophages to M1. The study by Sun et al (39) has shown that in rheumatoid arthritis, activation of the Notch pathway has the effect of activating macrophages and that the M1 macrophage was the most abundant cells with Notch activation. Notch signaling inhibitor Thapsigargin can attenuate joint tissue damage by switching M1 to M2 macrophages. It has also been found that in human Crohn's disease cells, inhibition of Notch signaling caused macrophages to polarize to $\mathrm{M} 2$, thereby reducing intestinal epithelial cell damage (43). In tumor cells, activation of the Notch pathway also causes macrophage activation and polarization to M1 $(38,44)$, indicating that in VILI rats, inhibition of Notch pathway could 
A NICD Hes-1

Hes-5

Hey1
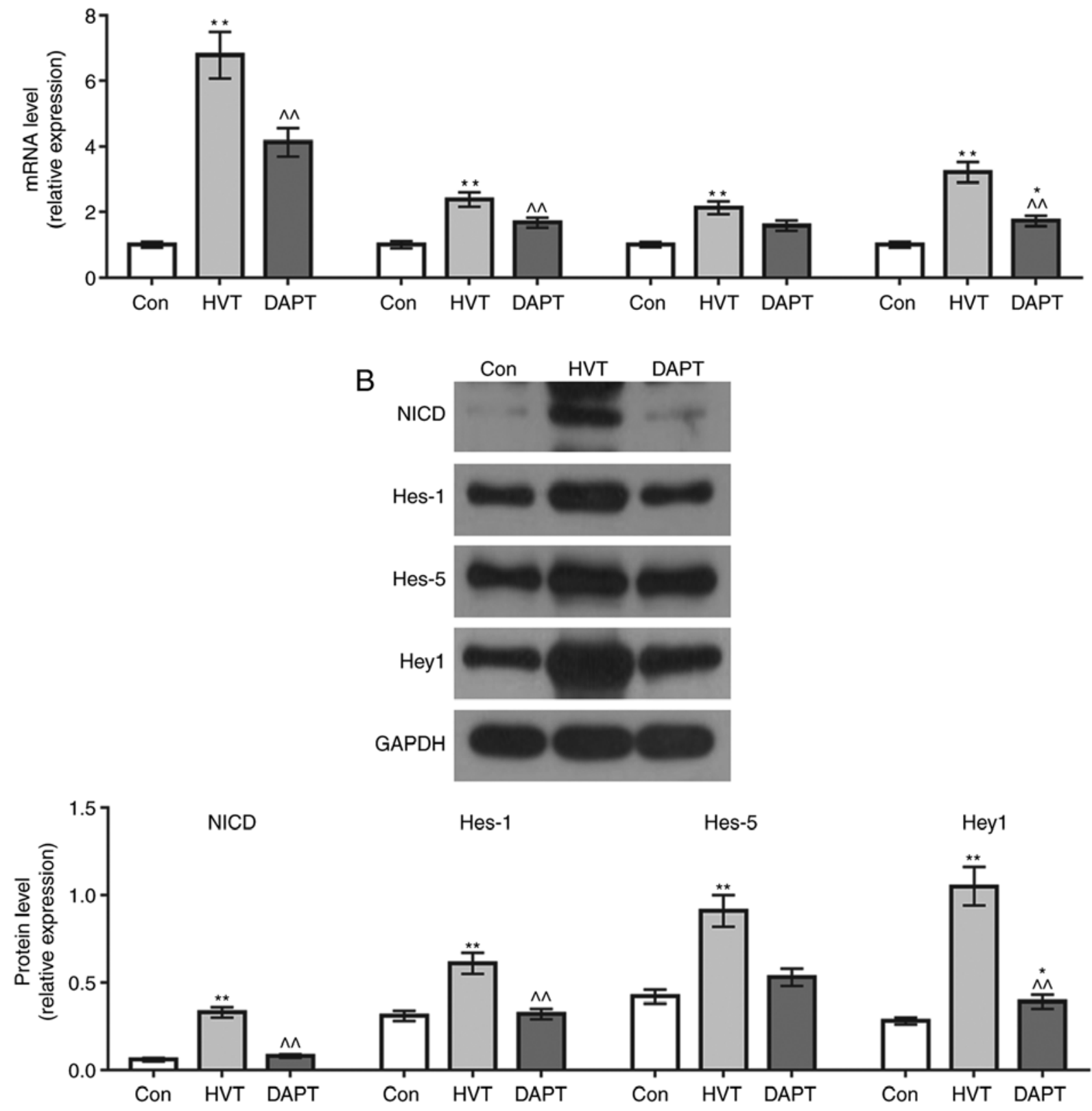

Figure 6. Effects of mechanical ventilation on the Notch pathway of macrophages. (A) The mRNA expression of the NICD, Hes1, Hes5 and Hey1 in the Con, HVT and DAPT group were detected using reverse transcription-quantitative PCR. (B) The protein expressions of the NICD, Hes1, Hes5 and Heyl in Con, HVT and DAPT group were detected by western blotting. ${ }^{*} \mathrm{P}<0.05$ and ${ }^{* *} \mathrm{P}<0.01$ vs. Con group; ${ }^{\wedge} \mathrm{P}<0.01$ vs. HVT group. HVT, high volume tidal; Con, control; DAPT, N-[N-(3,5-difluorophenylacetyl)-1-alanyl] phenylglycine t-butyl ester; NICD, Notch intracellular domain.

reduce inflammatory response by inhibiting $\mathrm{M} 1$ polarization of macrophages, therefore protecting lung tissue.

However, there were some limitations in the present study, for example, at multiple time points it was necessary to further explore the effects of different mechanical ventilation durations on rat lung tissue. In the current study, F4/80 and INOS were used to assess the M1 and M2 macrophages, however, using more macrophage markers to gate the macrophage and M1/M2 phenotype may help reach a more convincing conclusion. The issue will be further analyzed comprehensively. In the present study, the polarization levels of M1 and M2 macrophages were detected by flow cytometry, which should be confirmed by performing immunofluorescence analysis.
In conclusion, the present study demonstrated that high-frequency mechanical ventilation could cause inflammation and macrophages were polarized toward M1. The activation of the Notch pathway in macrophages was also found. After using DAPT to inhibit the Notch pathway, the lung damage caused by mechanical ventilation was reduced and the level of inflammatory factors was also reduced, which may be explained by that the inhibition of the Notch pathway could inhibit macrophage polarization to M1.

\section{Acknowledgements}

Not applicable. 


\section{Funding}

The present study was supported by the Dean's Fund of Jinan Military General Hospital in 2015 (grant. no. 2015QN02).

\section{Availability of data and materials}

The analyzed data sets generated during the present study are available from the corresponding author on reasonable request.

\section{Authors' contributions}

Substantial contributions to conception and design; data acquisition, data analysis and interpretation, drafting the article or critically revising it for important intellectual content, final approval of the version to be published and agreement to be accountable for all aspects of the work in ensuring that questions related to the accuracy or integrity of the work are appropriately investigated and resolved: All authors.

\section{Ethics approval and consent to participate}

All animal procedures were approved by the Institutional Animal Care and Use Committee and China Council on Animal Care. All animal experiments were performed in compliance with the Guidelines for Proper Conduct of Animal Experiments, established by the Science Council.

\section{Patient consent for publication}

Not applicable.

\section{Competing interests}

The authors declare that they have no competing interests.

\section{References}

1. Sutherasan Y, Vargas M and Pelosi P: Protective mechanical ventilation in the non-injured lung: Review and meta-analysis. Crit Care 18: 211, 2014.

2. Schnell D, Timsit JF, Darmon M, Vesin A, Goldgran-Toledano D, Dumenil AS, Garrouste-Orgeas M, Adrie C, Bouadma L, Planquette $\mathrm{B}$, et al: Noninvasive mechanical ventilation in acute respiratory failure: Trends in use and outcomes. Intensive Care Med 40: 582-591, 2014.

3. Unroe M, Kahn JM, Carson SS, Govert JA, Martinu T, Sathy SJ, Clay AS, Chia J, Gray A, Tulsky JA and Cox CE: One-year trajectories of care and resource utilization for recipients of prolonged mechanical ventilation: A cohort study. Ann Intern Med 153: 167-175, 2010.

4. Müller-Redetzky HC, Felten M, Hellwig K, Wienhold SM, Naujoks J, Opitz B, Kershaw O, Gruber AD, Suttorp N and Witzenrath M: Increasing the inspiratory time and I:E ratio during mechanical ventilation aggravates ventilator-induced lung injury in mice. Crit Care 19: 23, 2015.

5. Hamlington KL, Smith BJ, Allen GB and Bates JH: Predicting ventilator-induced lung injury using a lung injury cost function. J Appl Physiol (1985) 121: 106-114, 2016.

6. Reddy SP, Hassoun PM and Brower R: Redox imbalance and ventilator-induced lung injury. Antioxid Redox Signal 9: 2003-2012, 2007.

7. Curley GF, Contreras M, Higgins B, O'Kane C, McAuley DF, O'Toole D and Laffey JG: Evolution of the inflammatory and fibroproliferative responses during resolution and repair after ventilator-induced lung injury in the rat. Anesthesiology 115: 1022-1032, 2011.
8. Bertok S, Wilson MR, Morley PJ, de Wildt R, Bayliffe A and Takata M: Selective inhibition of intra-alveolar p55 TNF receptor attenuates ventilator-induced lung injury. Thorax 67: 244-251,2012.

9. Wilson MR, Wakabayashi K, Bertok S, Oakley CM, Patel BV, O'Dea KP, Cordy JC, Morley PJ, Bayliffe AI and Takata M: Inhibition of TNF receptor p55 By a domain antibody attenuates the initial phase of acid-induced lung injury in mice. Front Immunol 8: 128, 2017.

10. Opitz B, van Laak V, Eitel J and Suttorp N: Innate immune recognition in infectious and noninfectious diseases of the lung. Am J Respir Crit Care Med 181: 1294-1309, 2010.

11. Martinez FO and Gordon S: The M1 and M2 paradigm of macrophage activation: Time for reassessment. F1000Prime Rep 6: 13 , 2014.

12. Jablonski KA, Amici SA, Webb LM, Ruiz-Rosado Jde D, Popovich PG, Partida-Sanchez S and Guerau-de-Arellano M: Novel markers to delineate murine M1 and M2 macrophages. PLoS One 10: e0145342, 2015.

13. Wynn TA, Chawla A and Pollard JW: Macrophage biology in development, homeostasis and disease. Nature 496: 445-455, 2013.

14. Jaecklin T, Otulakowski G and Kavanagh BP: Do soluble mediators cause ventilator-induced lung injury and multi-organ failure? Intensive Care Med 36: 750-757, 2010.

15. Dai H, Pan L, Lin F, Ge W, Li W and He S: Mechanical ventilation modulates Toll-like receptors 2,4 , and 9 on alveolar macrophages in a ventilator-induced lung injury model. J Thorac Dis 7: 616-624, 2015.

16. Kopan R and Ilagan MX: The canonical Notch signaling pathway: Unfolding the activation mechanism. Cell 137: 216-233, 2009.

17. Yang Z, Guo L, Liu D, Sun L, Chen H, Deng Q, Liu Y, Yu M, Ma Y, Guo N and Shi M: Acquisition of resistance to trastuzumab in gastric cancer cells is associated with activation of IL-6/STAT3/Jagged-1/Notch positive feedback loop. Oncotarget 6: 5072-5087, 2015.

18. Han H, Gong G, Bai X, Lin YC, Sun J, Wang W, Zhao Y, Yang L, Wang X, Zhang Z, et al: Inhibition of Notch signaling protects mouse lung against zymosan-induced injury. Shock 40: 312-319, 2013.

19. Tsao PN, Wei SC, Huang MT, Lee MC, Chou HC, Chen CY and Hsieh WS: Lipopolysaccharide-induced Notch signaling activation through JNK-dependent pathway regulates inflammatory response. J Biomed Sci 18: 56, 2011.

20. Jiao Z, Wang W, Hua S, Liu M, Wang H, Wang X, Chen Y, Xu H and Lu L: Blockade of Notch signaling ameliorates murine collagen-induced arthritis via suppressing Th1 and Th17 cell responses. Am J Pathol 184: 1085-1093, 2014.

21. Park JS, Kim SH, Kim K, Jin CH, Choi KY, Jang J, Choi Y, Gwon AR, Baik SH, Yun UJ, et al: Inhibition of Notch signalling ameliorates experimental inflammatory arthritis. Ann Rheum Dis 74: 267-274, 2015.

22. Huang C, Pan L, Lin F, Dai H and Fu R: Monoclonal antibody against Toll-like receptor 4 attenuates ventilator-induced lung injury in rats by inhibiting MyD88- and NF- $\mathrm{KB}$-dependent signaling. Int J Mol Med 39: 693-700, 2017.

23. Whitehead TC, Zhang H, Mullen B and Slutsky AS: Effect of mechanical ventilation on cytokine response to intratracheal lipopolysaccharide. Anesthesiology 101: 52-58, 2004.

24. Livak KJ and Schmittgen TD: Analysis of relative gene expression data using real-time quantitative PCR and the 2(-Delta Delta C(T)) method. Methods 25: 402-408, 2001.

25. Silva PL, Negrini D and Rocco PR: Mechanisms of ventilator-induced lung injury in healthy lungs. Best Pract Res Clin Anaesthesiol 29: 301-313, 2015.

26. Cressoni M, Gotti M, Chiurazzi C, Massari D, Algieri I, Amini M, Cammaroto A, Brioni M, Montaruli C, Nikolla K, et al: Mechanical power and development of Ventilator-induced lung injury. Anesthesiology 124: 1100-1108, 2016.

27. Borges JB, Costa EL, Suarez-Sipmann F, Widström C, Larsson A, Amato M and Hedenstierna G: Early inflammation mainly affects normally and poorly aerated lung in experimental ventilator-induced lung injury*. Crit Care Med 42: e279-e287, 2014.

28. Xu YH and Guo NL: USP14 inhibitor IU1 prevents ventilatorinduced lung injury in rats. Cell Mol Biol (Noisy-le-grand) 60: 50-54, 2014.

29. Malaviya R, Sunil VR, Venosa A, Verissimo VL, Cervelli JA, Vayas KN, Hall L, Laskin JD and Laskin DL: Attenuation of nitrogen mustard-induced pulmonary injury and fibrosis by anti-tumor necrosis factor- $\alpha$ antibody. Toxicol Sci 148: 71-88, 2015. 
30. Halbertsma FJ, Vaneker M, Scheffer GJ and van der Hoeven JG: Cytokines and biotrauma in ventilator-induced lung injury: A critical review of the literature. Neth J Med 63: 382-392, 2005.

31. Ko YA, Yang MC, Huang HT, Hsu CM and Chen LW: NF-кB activation in myeloid cells mediates ventilator-induced lung injury. Respir Res 14: 69, 2013.

32. Huang C, Pan L, Lin F, Qian W and Li W: Alveolar macrophage TLR4/MyD88 signaling pathway contributes to ventilator-induced lung injury in rats. Xi Bao Yu Fen Zi Mian Yi Xue Za Zhi 31: 182-185, 189, 2015 (In Chinese).

33. Morimoto M, Nishinakamura R, Saga Y and Kopan R: Different assemblies of Notch receptors coordinate the distribution of the major bronchial Clara, ciliated and neuroendocrine cells. Development 139: 4365-4373, 2012.

34. Nagase $H$ and Nakayama $K: \gamma$-Secretase-regulated signaling typified by Notch signaling in the immune system. Curr Stem Cell Res Ther 8: 341-356, 2013.

35. Katoh $\mathrm{M}$ and Katoh $\mathrm{M}$ : Integrative genomic analyses on HES/HEY family: Notch-independent HES1, HES3 transcription in undifferentiated ES cells, and Notch-dependent HES1, HES5, HEY1, HEY2, HEYL transcription in fetal tissues, adult tissues, or cancer. Int J Oncol 31: 461-466, 2007.

36. Ma Y, Bian J and Zhang F: Inhibition of perillyl alcohol on cell invasion and migration depends on the Notch signaling pathway in hepatoma cells. Mol Cell Biochem 411: 307-315, 2016.

37. Liu X, Cong N, Cheng X, Ma R, Wang J, Huang YB, Zhao M, Wang XW, Chi FL and Ren DD: The role of the Notch signal pathway in mucosal cell metaplasia in mouse acute otitis media. Sci Rep 7: 4588, 2017.

38. Wang YC, He F, Feng F, Liu XW, Dong GY, Qin HY, Hu XB, Zheng $\mathrm{MH}$, Liang L, Feng L, et al: Notch signaling determines the M1 versus M2 polarization of macrophages in antitumor immune responses. Cancer Res 70: 4840-4849, 2010.
39. Sun W, Zhang H, Wang H, Chiu YG, Wang M, Ritchlin CT, Kiernan A, Boyce BF and Xing L: Targeting notch-activated M1 macrophages attenuates joint tissue damage in a mouse model of inflammatory arthritis. J Bone Miner Res 32: 1469-1480, 2017.

40. Huang F, Zhao JL, Wang L, Gao CC, Liang SQ, An DJ, Bai J, Chen Y, Han H and Qin HY: miR-148a-3p mediates Notch signaling to promote the differentiation and M1 activation of macrophages. Front Immunol 8: 1327, 2017.

41. Wongchana $\mathrm{W}$ and Palaga T: Direct regulation of interleukin-6 expression by Notch signaling in macrophages. Cell Mol Immunol 9: 155-162, 2012.

42. Zhang H, Hilton MJ, Anolik JH, Welle SL, Zhao C, Yao Z, Li X, Wang Z, Boyce BF and Xing L: NOTCH inhibits osteoblast formation in inflammatory arthritis via noncanonical NF- $\kappa \mathrm{B}$. J Clin Invest 124: 3200-3214, 2014.

43. Ortiz-Masia D, Cosin-Roger J, Calatayud S, Hernández C, Alós R, Hinojosa J, Esplugues JV and Barrachina MD: M1 macrophages activate Notch signalling in epithelial cells: Relevance in crohn's disease. J Crohns Colitis 10: 582-592, 2016.

44. Zhao JL, Huang F, He F, Gao CC, Liang SQ, Ma PF, Dong GY, Han H and Qin HY: Forced activation of Notch in macrophages represses tumor growth by upregulating miR-125a and disabling tumor-associated macrophages. Cancer Res 76: 1403-1415, 2016.

This work is licensed under a Creative Commons Attribution-NonCommercial-NoDerivatives 4.0 International (CC BY-NC-ND 4.0) License. 